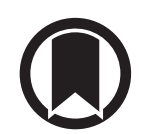

CrossMark

\title{
Can circular RNAs be used as prenatal biomarkers for congenital diaphragmatic hernia?
}

\author{
To the Editor:
}

Since 2000, more than 400000 babies worldwide have died of congenital diaphragmatic hernia (CDH), a condition that is occurring as frequently as cystic fibrosis and characterised by underdeveloped lungs (pulmonary hypoplasia), persistent pulmonary hypertension and a diaphragmatic defect [1]. CDH can be diagnosed prenatally with ultrasound and fetal MRI, but outcome prediction and diagnostic accuracy remain imperfect [2]. The observed over expected lung-to-head (O/E LHR) ratio at 22-23 and 32-33 weeks of gestation is currently used to predict CDH outcomes [3]. A prenatal biomarker for the assessment of disease severity and prognostication has not been established yet. In contrast to cystic fibrosis, a common genetic cause has not been identified for $\mathrm{CDH}$, suggesting that epigenetic and environmental factors are involved in the pathogenesis. We have previously discovered that microRNA $200 \mathrm{~b}$ (miR-200b) is highly dysregulated in hypoplastic human CDH lungs and that miR-200b administration can serve as a prenatal therapy in an animal model for $\mathrm{CDH}[4,5]$.

Circular RNAs (circRNAs) are powerful upstream regulators of microRNAs (miRNAs). They arise from alternative splicing events of pre-mRNA where the ends are ligated together to form a circle. Their expression is organ and development specific [6]. Due to their shape, they are highly stable in body fluids (e.g. blood) and thought to be ideal biomarkers [7]. Consequently, circRNAs are under investigation as disease specific markers in different cancers and cardiovascular disease [8,9]. We hypothesised that the circRNA profile of fetal hypoplastic $\mathrm{CDH}$ lungs is significantly dysregulated compared with lungs from controls and that they can serve as future prenatal biomarkers for the improved outcome prediction of $\mathrm{CDH}$. Our objective for this preliminary study was to profile and compare circRNAs in human fetal control and $\mathrm{CDH}$ lungs at two different timepoints of fetal development (mid- and end-pregnancy) to identify a profile of circRNAs that can guide a future clinical trial to test the first ever non-invasive biomarker for prenatal prognostication of $\mathrm{CDH}$.

After institutional review board approval (HS15293 (H2012:134)) and written consent from the parents, we used formalin-fixed, paraffin-embedded lung tissue blocks from autopsies of severe CDH cases $(n=6)$ without associated anomalies and age-matched controls $(n=6)$ from a previously established biobank [10]. No standardised genetic testing was performed at our institution at the time tissues were obtained. Lung tissues were derived from deceased patients at two different gestational ages (GA) ( 20 weeks $\mathrm{GA}=$ mid-pregnancy, versus $\sim 40$ weeks $\mathrm{GA}=\mathrm{end}$ pregnancy). Control subjects died from a condition unrelated to lung disease (e.g. chorioamnionitis, placental abruption or placental vasculopathologies), which was confirmed by independent pathological examination. Pulmonary hypoplasia was assessed post mortem and total lung weight to head circumference ratios were used to measure the degree of lung hypoplasia. $\mathrm{CDH}$ patients had lung weight to head circumference ratios of $0.25 \pm 0.07$ and $0.99 \pm 0.32$, whereas control patients had ratios of $0.45 \pm 0.09$ and $2.43 \pm 0.91$ for mid- and end-gestation, respectively. Using established protocols, RNA extraction was performed with an RNA isolation kit (Exiqon, Woburn, MA, USA). RNA concentration was determined using a NanoDrop 1000 spectrophotometer (Thermo Scientific, Wilmington, DE, USA) and RNA integrity was assessed by electrophoresis on a denaturing agarose gel. Microarray was performed based on a previously established standard protocol (Arraystar, Rockville, MD, USA). After RNase R (Epicentre, Inc.) enrichment, circRNAs were amplified and

@ERSpublications

Circular RNAs are dysregulated in lungs of congenital diaphragmatic hernia patients, a malformation of the lung and diaphragm. These results suggest that they can serve as prenatal biomarkers to improve prognostication and diagnostic accuracy. http://bit.ly/2Cz7Bzm

Cite this article as: Wagner R, Jha A, Ayoub L, et al. Can circular RNAs be used as prenatal biomarkers for congenital diaphragmatic hernia? Eur Respir J 2020; 55: 1900514 [https://doi.org/10.1183/ 13993003.00514-2019]. 

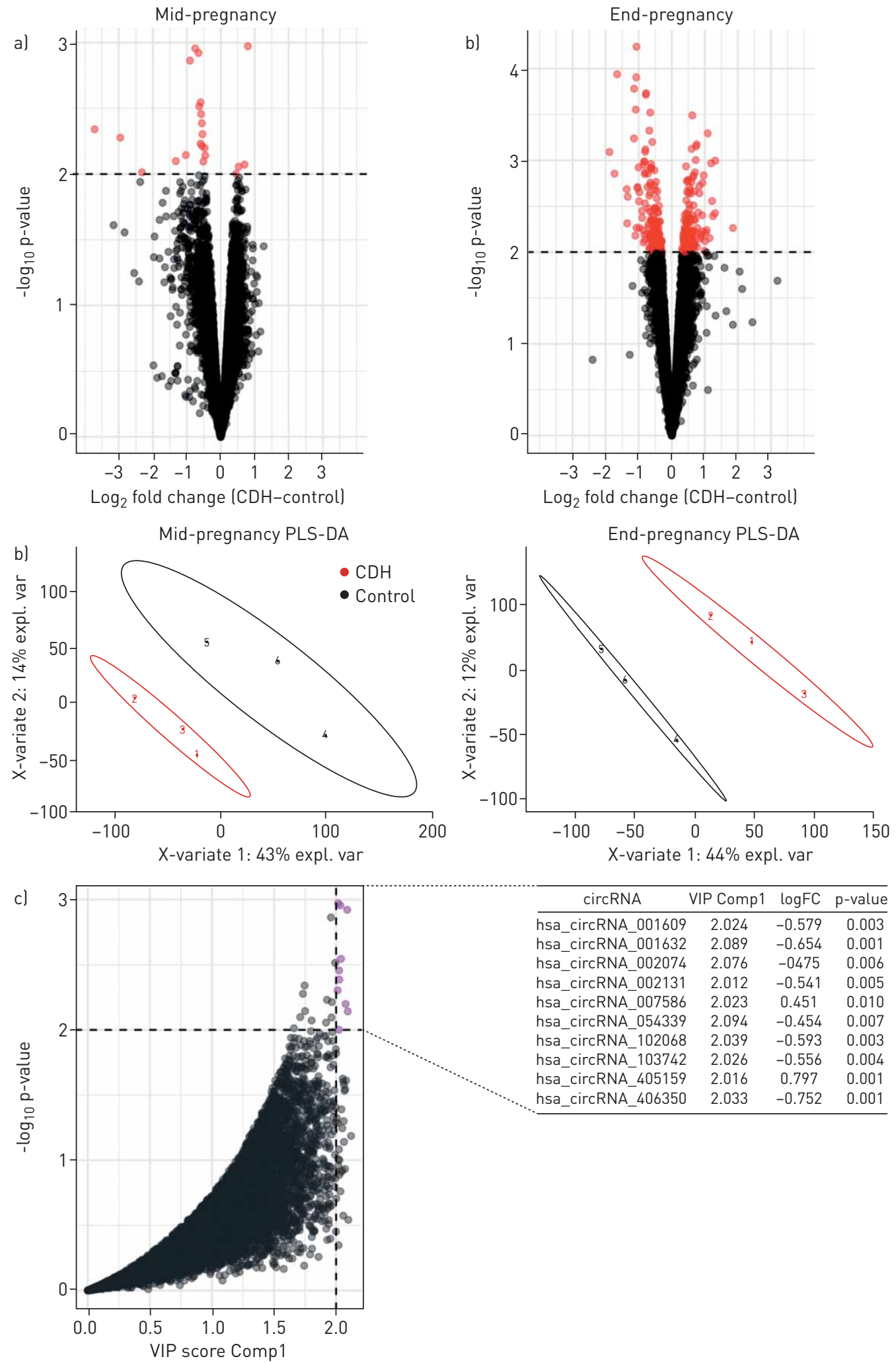

FIGURE 1 Circular RNA profile is highly dysregulated in human fetal congenital diaphragmatic hernia (CDH) lungs compared with controls and can distinguish CDH from control tissues. a) Volcano plot for early and late stages of lung development indicating a dysregulated circRNA profile in CDH lungs. Dotted horizontal line at $p$-value $<0.01$. b) Partial least squares discriminant analysis of the microarray expression values shows that CDH and control lungs cluster strongly into independent groups ( $n=3$ per group). c) Cluster plot with variable importance in projection (VIP)-score (VIP $\geqslant 2$ ) and p-value $(<0.01)$ identifies 10 circRNAs with the highest significant change in expression. The table shows a list of names of the 10 top targets together with their VIP score, fold change (FC) and p-value. COMP1: compartment 1; PLS-DA: partial least squares discriminant analysis; expl. var: explained variable. 
transcribed into fluorescent cRNA. Subsequent hybridisation of the labelled cRNAs onto the Array $(8 \times 15 \mathrm{~K}$, Arraystar $)$ was followed by washing steps. Arrays were then scanned with an Agilent Scanner (G2505C). Quantile normalisation and data processing were performed with R software (limma and ggplot packages) $[11,12]$. To mitigate the effects of our limited sample size, we used a more stringent cut off at $\mathrm{p}<0.01$.

A total of 10140 and 12055 circRNAs were detected for mid- and end-pregnancy cases, respectively. From these, comparing $\mathrm{CDH}$ and age-matched control lungs, we identified significant $(\mathrm{p}<0.01)$ alterations in 22 circRNAs at mid-pregnancy and 281 at end pregnancy between CDH lungs and age-matched controls (figure 1a). We performed a partial least squares discriminant analysis (PLS-DA) and showed that CDH lungs formed a separate cluster from controls, based on their circRNA expression profile (figure 1b). This indicates that the circRNA profile can discriminate the CDH lungs from controls. To identify potential biomarkers that can be used earlier during pregnancy, we focused on mid-pregnancy cases for further analysis. We used variable importance in projection (VIP) scores obtained from our PLS-DA to determine the most important contributors to the segregation between $\mathrm{CDH}$ and control. This revealed 5064 circRNAs with a VIP score greater than 1, which are considered important in the variable separation by PLS-DA. Due to the limited number of fetal $\mathrm{CDH}$ lungs in this study, we applied a more stringent selection criterium to identify potential circRNA biomarkers by combining the VIP score analysis and $\mathrm{p}$-values obtained from the differential expression analysis (VIP score $\geqslant 2$ and $p$-value $<0.01$ ). Using these criteria, we identified 10 circRNAs that were most important in defining the CDH lung signature compared to healthy control lungs (figure 1c).

We have previously identified dysregulation of miRNAs in fetal lungs and lung fluid of CDH babies [4]. CircRNAs are potent regulators of miRNAs and have not been investigated in abnormal fetal lung development and as potential biomarkers for $\mathrm{CDH}$. This is the first study to identify an altered expression profile of circRNAs in a small sample of hypoplastic $\mathrm{CDH}$ lungs at two different time points of fetal lung development. We show that circRNA profiles are changed in lungs from $\mathrm{CDH}$ fetuses compared to control lungs. Moreover, using PLS-DA analysis, we determined that the $\mathrm{CDH}$ lungs form a separate cluster from control lungs based on their circRNA expression values. Using a novel bioinformatic approach, we identified 10 circRNAs from mid-pregnancy cases that can inform future biomarker verification and validation studies. Validation of these results in a prospective and independent cohort is required to confirm our findings but this study provides the necessary preliminary work to design and execute such a study. Due to the limited number of $\mathrm{CDH}$ cases in a single centre, a multi-institutional approach will be required to obtain a sufficient sample size in a clinically relevant cohort.

To date, prenatal diagnosis and outcome prediction of $\mathrm{CDH}$ relies entirely on imaging modalities with limited sensitivity and imperfect prognostication methods [2]. No minimally invasive biomarker from maternal body fluids exists for $\mathrm{CDH}$ [13]. Others have shown that long non-coding RNAs can serve as biomarkers for the prenatal diagnosis of fetal heart defects analysing maternal plasma but we have yet to apply this technology to congenital lung abnormalities [14]. CircRNAs are development specific, organspecific and robust in plasma due to their circular structure making them resistant to endonucleases [15]. Their characteristics together with our results highlight their potential as prenatal non-invasive biomarkers for better severity assessment of $\mathrm{CDH}$. Therefore, future studies will include circRNA analysis in maternal body fluids during pregnancy. Here, we discovered a circRNA profile distinguishing CDH lungs from control lungs. However, our experimental settings and the inherent limitations do not allow separation from other lung pathologies at this point. Analysis of circRNA profiles in a larger cohort will allow linkage of a specific circRNA signature to clinical outcomes and severity of the individual CDH cases. It will also be important to determine if $\mathrm{CDH}$ patients with associated malformations and chromosomal abnormalities have a specific circRNA profile. This will allow further development of a circRNA biomarker panel that can potentially assist with prenatal prognostication and counselling. Point-of-care diagnostic measures using biomarkers together with current image-based modalities will likely improve the outcome prediction of $\mathrm{CDH}$ globally.

Richard Wagner $\odot^{1,2,3,4}$, Aruni Jha ${ }^{2,3}$, Lojine Ayoub ${ }^{1,2,3}$, Shana Kahnamoui ${ }^{1,2,3}$, Daywin Patel ${ }^{1,3}$, Thomas H. Mahood ${ }^{2,3}$, Andrew J. Halayko ${ }^{2,3}$, Martin Lacher ${ }^{4}$, Christopher D. Pascoe $\circledast^{2,3}$ and Richard Keijzer $\circledast^{1,2,3}$ ${ }^{1}$ Depts of Surgery, Division of Pediatric Surgery, Pediatrics \& Child Health and Physiology \& Pathophysiology (Adjunct), University of Manitoba, Winnipeg, MB, Canada. ${ }^{2}$ Dept of Physiology and Pathophysiology, University of Manitoba, Winnipeg, MB, Canada. ${ }^{3}$ Biology of Breathing Theme, Children's Hospital Research Institute of Manitoba, Winnipeg, MB, Canada. ${ }^{4}$ Dept of Pediatric Surgery, University Hospital Leipzig, Leipzig, Germany.

Correspondence: Richard Keijzer, Dept of Surgery, Division of Pediatric Surgery, University of Manitoba and Children's Hospital Research Institute of Manitoba, AE402-840 Sherbrook Street Winnipeg, Manitoba, Canada, R3A 1R9. E-mail: richard.keijzer@umanitoba.ca 
Received: 11 March 2019 | Accepted after revision: 29 Oct 2019

Support statement: This research was supported by funds to R. Keijzer from the Canadian Institutes of Health Research and the Biology of Breathing Theme of the Children's Hospital Research Institute of Manitoba. R. Keijzer is the recipient of a Career Enhancement Award from the Canadian Child Health Clinician Scientist Program and a New Investigator Salary Award from the Canadian Institutes of Health Research, Manitoba Lung Association and the Children's Hospital Research Institute of Manitoba. The content is solely the responsibility of the authors and does not represent the official views of the CIHR. Funding information for this article has been deposited with the Crossref Funder Registry.

Conflict of interest: None declared.

\section{References}

$1 \quad$ Keijzer R, Puri P. Congenital diaphragmatic hernia. Semin Pediatr Surg 2010; 19: 180-185.

2 Burgos CM, Frenckner B, Luco M, et al. Prenatally versus postnatally diagnosed congenital diaphragmatic hernia Side, stage, and outcome. J Pediatr Surg 2019; 54: 651-655.

3 Jani J, Nicolaides KH, Benachi A, et al. Timing of lung size assessment in the prediction of survival in fetuses with diaphragmatic hernia. Ultrasound Obstet Gynecol 2008; 31: 37-40.

4 Pereira-Terra P, Deprest JA, Kholdebarin R, et al. Unique tracheal fluid MicroRNA signature predicts response to FETO in patients with congenital diaphragmatic hernia. Ann Surg 2015; 262: 1130-1140.

5 Khoshgoo N, Kholdebarin R, Pereira-Terra P, et al. Prenatal microRNA miR-200b therapy improves Nitrofen-induced pulmonary hypoplasia associated with congenital diaphragmatic hernia. Ann Surg 2019; 269: 979-987.

6 Haque S, Harries LW. Circular RNAs (CircRNAs) in health and disease. Genes 2017; 8: E353.

$7 \quad$ Abu N, Jamal R. Circular RNAs as promising biomarkers: A mini-review. Front Physiol 2016; 7: 355.

8 Kristensen LS, Hansen TB, Venø MT, et al. Circular RNAs in cancer: opportunities and challenges in the field. Oncogene 2018; 37: 555-565.

9 Zhao Z, Li X, Gao C, et al. Peripheral blood circular RNA hsa_circ_0124644 can be used as a diagnostic biomarker of coronary artery disease. Sci Rep 2017; 7: 39918.

10 Wagner R, Ayoub L, Kahnamoui S, et al. Establishment of a biobank for human lung tissues of congenital diaphragmatic hernia and congenital pulmonary airway malformation. J Pediatr Surg 2019; 54: 2439-2442.

11 Phipson B, Lee S, Majewski IJ, et al. Robust hyperparameter estimation protects against hypervariable genes and improves power to detect differential expression. Ann Appl Stat 2016; 10: 946-963.

12 Wickham H. ggplot2: Elegant Graphics for Data Analysis. New York, Springer-Verlag, 2016

13 Wagner R, Hei Tse W, Gosemann J-H, et al. Prenatal maternal biomarkers for the early diagnosis of congenital malformations: A review. Pediatr Res 2019; 86: 560-566.

$14 \mathrm{Gu} \mathrm{M}$, Zheng $\mathrm{A}, \mathrm{Tu} \mathrm{W}$, et al. Circulating LncRNAs as novel, non-invasive biomarkers for prenatal detection of fetal congenital heart defects. Cell Physiol Biochem 2016; 38: 1459-1471.

15 Hansen TB, Jensen TI, Clausen BH, et al. Natural RNA circles function as efficient microRNA sponges. Nature 2013; 495: 384-388. 